\title{
Multipath Characteristics for Mobile to Mobile Direct Communications based on Channel Measurement in Urban Street Grid Environments
}

\author{
Myung-Don KIM, Juyul LEE, Jinyi LIANG and Jinup KIM \\ Electronics and Telecommunications Research Institute (ETRI) \\ 218, Gajeongno, Yuseong-gu, Daejeon, 305-700, Korea \\ \{mdkim, jujul, liangjinyi, jukim\}@etri.re.kr
}

\begin{abstract}
In this paper, we focus on multipath channel characteristics of low-height antenna links for mobile to mobile communications in urban street grid environments. We present a wideband MIMO channel sounder and antennas used to measure multipath channel characteristics at $3.7 \mathrm{GHz}$ frequency band and the result of calibration test to evaluate a system performance before field measurement. We carried out a channel measurement campaign in urban street grid environments in Seoul, Korea. The results of multipath characteristics from collected data in field measurement are compared with the conventional ITU-R channel model parameters in terms of r.m.s delay spread, and angular spread of arrival and departure.
\end{abstract}

Keywords - Mobile-to-Mobile, multipath characteristics, channel model, channel measurements, channel sounder

\section{INTRODUCTION}

Mobile-to-mobile direct communication services, commonly known as D2D (device-to-device), are now being actively discussed in various standardization bodies, e.g., "ProSe" for the LTE-Advanced system [1]. Unlike typical rooftop cellular networks, both transmitters and receivers are generally found near street levels, since direct communication links are established between mobile terminals. Consequently, conventional propagation models are limited in their ability to predict specific channel environments and those characteristics for development of the direct communication system using a system or link level channel simulation.

In this paper, we focus on channel characteristics of low antenna links especially in rectangular street grid environments. Lu et al [2] developed simplified site-specific path loss formulas for street grids. They assumed that surrounding buildings are infinitely high so that "vertical plane" effects can be ignored. Considering reflection and diffraction along with the horizontal plane, they developed path loss formula based on two-ray models, which can account for up to two-turn NLoS links. Refer to [3], we also investigated the effects of surrounding building heights on path loss characteristics, especially in urban street grid environments in Korea. From an analysis of the measurements, we have discussed the effects of average building heights on path loss, comparing them with the ITU-R propagation models such as ITU-R Recommendation P.1411 [4] and Report M.2135 [5]. Interestingly, better propagation characteristics are measured where the average building height is higher. Furthermore, from the aspect of radio propagation between low-antenna terminals in high-rise environments, reflected waves at corners in horizontal plane seem to play a dominant role.

This paper discusses multipath characteristics for mobileto-mobile direct communications based on channel measurements in Seoul, Korea. We focus on multipath characteristics of low-height antenna links in rectangular street grid environments. Section II presents a wideband MIMO channel sounder and antennas used to measure multipath channel characteristics considering to mobile-tomobile direct communication service. We also present the result of calibration measurement to evaluate a system performance before field measurement. In Section III and Section IV, the field measurement campaign and analysis results of multipath characteristics such as angular azimuth spectrum, delay spread, and angular spread, in street grid environment, are described. Finally, to wrap up the work, conclusions are given in Section V.
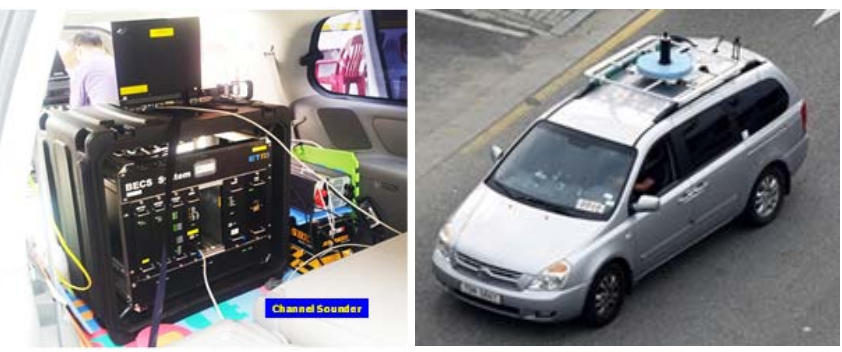

Figure 1. Channel sounder system and measurement antenna installation on the rooftop of vehicle

\section{Measurement System AND Calibration of System PERFORMANCE BEFORE MEASUREMENT}

\section{A. Wideband MIMO Channel Sounder and Antennas}

Measurement campaigns were conducted with the wideband MIMO channel sounder system developed at ETRI, 
which can measure multipath characteristics at carrier frequency $3.705 \mathrm{GHz}$ with $100 \mathrm{MHz}$ bandwidth (see Table 1).

TABLE 1. SPECIFICATION OF CHANNEL SOUNDER AND MEASUREMENT CONFIGURATIONS

\begin{tabular}{|l|l|}
\hline Characteristics & Measurement configurations \\
\hline \hline Center Frequency & $3.705 \mathrm{GHz}$ \\
\hline Bandwidth & $100 \mathrm{MHz}$ \\
\hline PN Code Length & 2048 chips \\
\hline Chip rate & $400 \mathrm{Mcps}$ \\
\hline RX ADC & $\begin{array}{l}\text { Sampling }: 400 \mathrm{MSa} / \mathrm{s} \\
\text { Resolution }: 12 \mathrm{bits}\end{array}$ \\
\hline TX Output Power (max.) & $+36 \mathrm{dBm}$ \\
\hline Multipath Resolution & $10 \mathrm{~ns}(3 \mathrm{~m})$ \\
\hline Number of Antenna elements & $\mathrm{TX}: 8, \mathrm{RX}: 16$ \\
\hline
\end{tabular}

Basically, the system can measure total $128(=8 \times 16)$ multiple channels in a time division multiplexing (TDM) fashion, i.e., multiple receive antennas sequentially receive sounding signals from multiple transmit antennas. To accomplish the acquisition of the signal data, the sounder system extracts channel impulse responses by exploiting the auto-correlation properties of pseudo-noise (PN) code [6][7].

TABLE 2. ANTENNA ARRAYS USED FOR MEASUREMENT

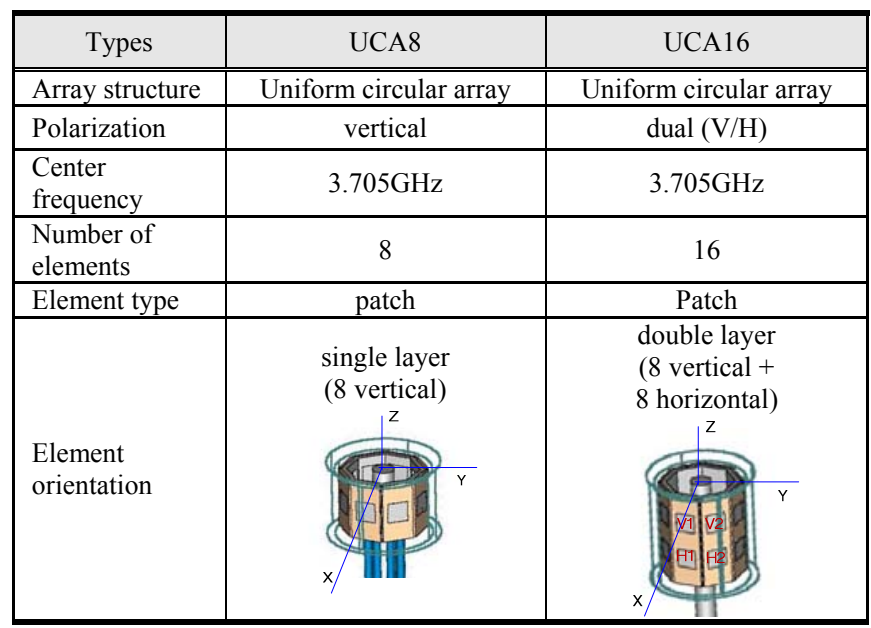

For the sake of convenience, we equipped both transmitter and receiver in separate vehicles (Figure 1), so that the transmitter and the receiver unit could be easily located anywhere along the traveling route. As shown in Figure 1, we installed uniform circular array (UCA) antennas on the rooftop of each vehicle, with the height of the antennas fixed at 1.9 meters. Table 2 shows an overview of the antenna arrays used in our measurement. UCA8 is a uniform circular array antenna set which has eight vertically polarized elements aligned circularly. UCA16 is a uniform circular array antenna which has double layered antenna elements. Eight vertically polarized elements make a circle for 360 degrees in the upper layer, and lower layer is consisted of eight horizontally polarized elements. All element types are patch type, and diameter of UCA16 and UCA 8 is $\lambda$. Inter-layer distance of $\mathrm{UCA} 8$ and UCA16 is $\lambda / 2$.

\section{B. System Calibration Measurement and Results}

Before measurement campaign, we have conducted a system calibration measurement at ETRI playground (open space) with channel sounders (a transmitter and a receiver) and antenna arrays. For this measurement, full radiation patterns of all the elements of each array antenna were measured in the anechoic chamber in advance. The azimuth plane radiation pattern with two polarizations (vertically and horizontally from $-180^{\circ}$ to $+180^{\circ}$ ) and the elevation plane (from $-45^{\circ}$ to $+45^{\circ}$ ) have been obtained enabling the estimation of the spatial channel characteristics. Figure 2 depicts a configuration of this test. The position of a transmitter (TX) was fixed, and a receiver (RX) was located at $9 \mathrm{~m}$ away. The UCA 8 array antenna for TX was installed on the ground with a height of $1.5 \mathrm{~m}$, and the UCA16 for RX was also positioned with a same height as a TX. We measured a channel from TX to RX, and changed the position of the RX and collected measured samples of total 8 points with different angles $\left(\theta=0^{\circ}, 45^{\circ}, 90^{\circ}, 135^{\circ}, 180^{\circ},-45^{\circ},-90^{\circ},-135^{\circ}\right)$.

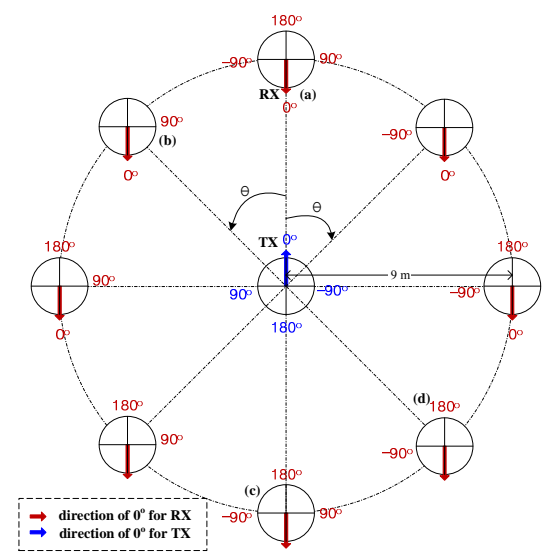

Figure 2. Configuration of calibration measurement for channel sounders (TX-RX) and antennas

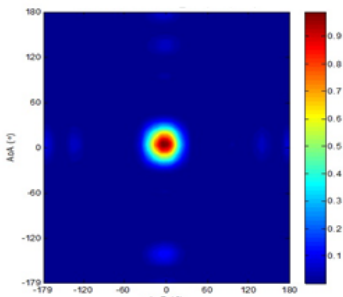

(a) $\theta=0^{\circ}$

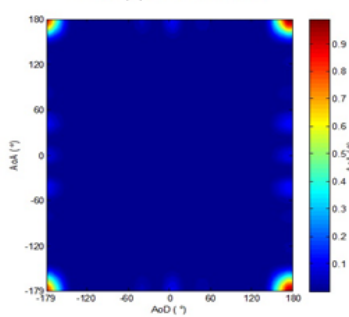

(c) $\theta=180^{\circ}$

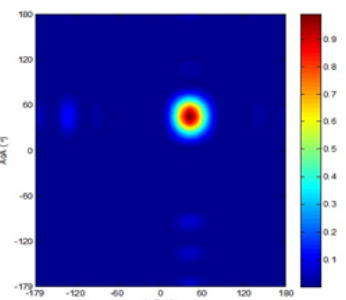

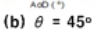

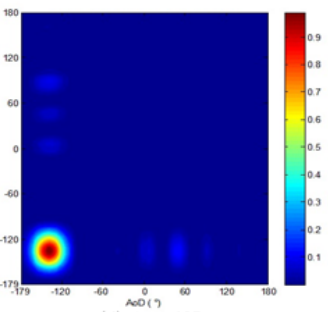

(d) $\theta=-135^{\circ}$
Figure 3. Estimated result of $A o A$ and $A o D$ at the position of $\theta$

In this paper, we observed the estimated results of an angle of departure (AoD) and an angle of arrival (AoA) by using a 
Barlett beam-forming method [3]. Figure 3 shows estimated results of AoA and AoD at each point of $\theta$. We can see that a dominant path component having the strongest channel power (red part) starts from each angle of $0^{\circ}, 45^{\circ}, 180^{\circ}$ and $-135^{\circ}$ and correctly arrives at each angle of $0^{\circ}, 45^{\circ}, 180^{\circ}$ and $-135^{\circ}$ in Figure 3(a)-(d). From this measurement results, the performance of our channel sounders was evaluated, and we confirmed the ability to estimate time and spatial characteristics of multipath channels through field measurement campaign.

\section{MEASUREMENT CAMPAigN}

To obtain multipath characteristics in urban street grid environments, we conducted a measurement campaign at Yeouido, Seoul in Korea. This measurement site is a commercial area with over 10 story buildings (35-40 meters height) and 7-12 meters wide streets. Figure 4 illustrates a layout of the site and surrounding environment, where high building blocks are aligned in a grid form. The channel sounder for TX is carried in a vehicle, and the UCA 8 antenna is mounted over the rooftop of the vehicle. The UCA16 antenna is mounted over the rooftop of a vehicle where the RX channel sounder installed on the inside. The height of each antenna for TX and RX is $1.9 \mathrm{~m}$ from the ground. We held TX at position $\bigcirc$ (yellow) in Figure 4, while RX is moving along with the routes R1, R2 and R3 respectively started from the position of TX. The RX gathers channel measurement data along the planned routes.

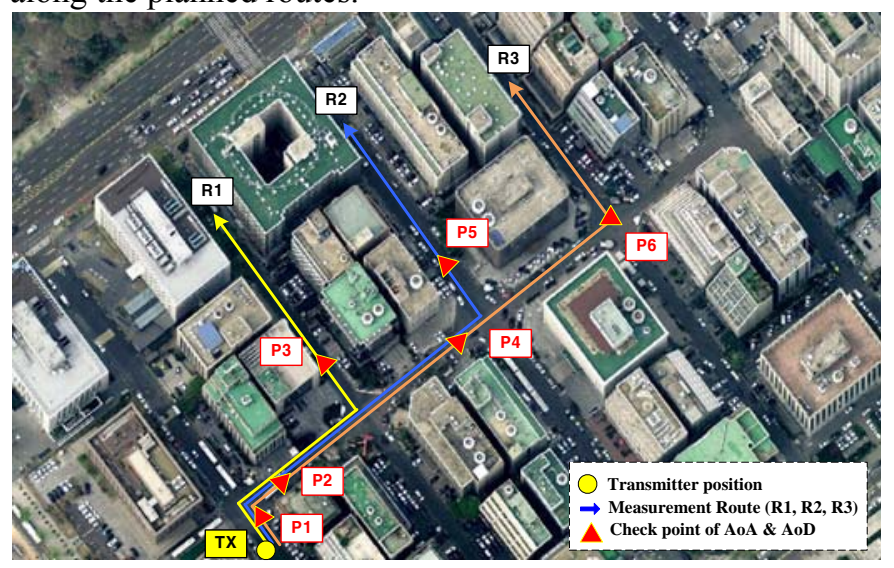

Figure 4. Example of an image with acceptable resolution

From this measurement campaign, the RX collected total 25,728 experimental samples $(=201$ points $\mathrm{x} 128$ channel impulse responses) at 201 points along with three travel routes. The measured data contains the raw baseband data for analysis of multipath channel characteristics and all GPS position information of the RXs.

\section{IV.Measurement Results AND ANALysis}

We analyzed multipath channel characteristics such as power azimuth spectrum (PAS), r.m.s delay spread (DS), angular spread of arrival (ASA) and angular spread of departure (ASD) from measured results.

\section{A. Power Azimuth Spectrum (PAS)}

The PAS calculation function is based on Bartlett beamforming theory [8], which is written as follows:

$$
\operatorname{PAS}\left(\varphi_{\mathrm{AOA}}\right)=\left|\sum_{\mathrm{k}}^{\mathrm{K}} \sum_{\mathrm{n}=1}^{\mathrm{N}} \mathrm{C}_{\mathrm{Rx}, \mathrm{n}}\left(\varphi_{\mathrm{AOA}}\right)^{\mathrm{H}} \cdot \mathrm{CIR}_{\mathrm{k}}\right|^{2}
$$

, where $\mathrm{K}$ is the length of $\mathrm{PN}$ sequence, $\mathrm{N}$ is the number of RX antenna elements, $C_{R x, n}\left(\varphi_{A O A}\right)$ is a radiation pattern of $\mathrm{RX}$, and $\varphi_{A O A}$ is an angle of arrival. And CIR denotes the channel impulse response which is from the auto/crosscorrelation function between an original PN sequence and a received signal as follows:

$$
\operatorname{CIR}(\tau)=\frac{R_{x y}(\tau)}{R_{x x}(0)}=\frac{\mathcal{F}^{-1}\left(-X(f)^{*} \cdot Y(f)\right)}{\sum_{i}\left|x\left(\tau_{i}\right)\right|^{2}}
$$

, where $Y(f)$ is a Fourier transform of the received signal $y(\tau), x(\tau)$ is a sequence for transmission, $R_{x x}$ means an autocorrelation to remove a system impairment [9], and $R_{x y}$ means a cross-correlation of $x(\tau)$ and $y(\tau)$.

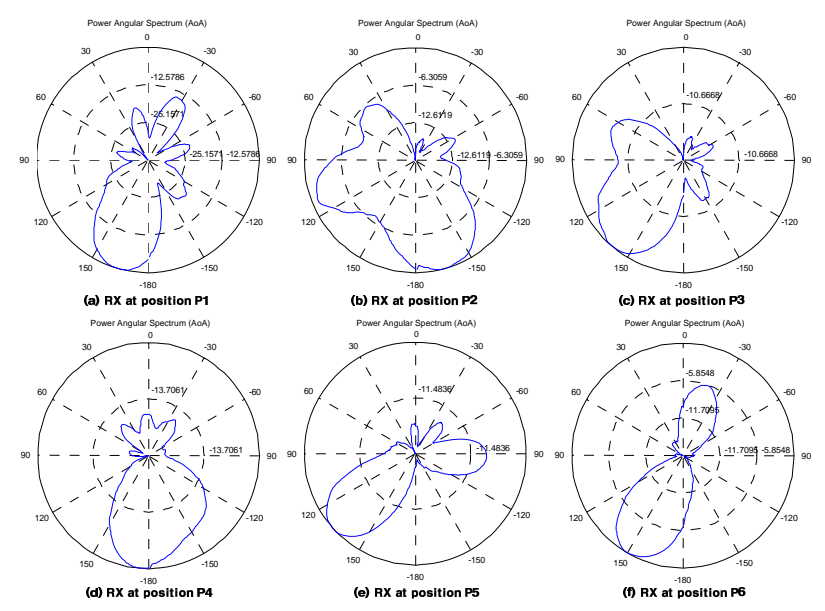

Figure 5. Measured results of power azimuth spectrum at each check points $(\mathrm{P} 1 \sim \mathrm{P} 6)$ in Figure 4

Figure 5 illustrate measured results of PAS at RX positions $\triangle(\mathrm{P} 1 \sim \mathrm{P} 6)$ as shown in Figure 4. We summarize the impinging multipath characteristics and directions (angles) at each point as follows,

- P1: Line of sight (LoS), 13m away from TX, receives a dominant path at $165^{\circ}$ directly from TX located at the rear

- P2: Non line of sight (NLoS), 10m after the first corner $(20 \mathrm{~m})$, receives a dominant path at $-165^{\circ}$ and paths at $110^{\circ}$ and $45^{\circ}$ reflected from buildings at corner area

- P3: NLoS, at the first block corner $(110 \mathrm{~m})$, receives a dominant path at $136^{\circ}$ from left road and paths at $65^{\circ}$ reflected from building

- P4: NLoS, at $98 \mathrm{~m}$ from the first corner, receives a dominant path at $180^{\circ}$ backside and spreads paths through the road

- P5: NLoS, after the second block corner (141m, R2), receives a dominant path at $130^{\circ}$ from left road and 
building, and a reflected wave at $-90^{\circ}$ from a right-side building

- P6: NLoS, at the third block corner (230m, R3), receives a dominant path at $150^{\circ}$ through the road and a reflected path at $-20^{\circ}$ from a building in the corner.

It can be observed from these results that a dominant path group is mostly appeared through the road when the RX is not located at corner area. At and after corner area, we can see that more multipath components are received by reflection from the adjacent buildings.

\section{B. r.m.s Delay Spread (DS)}

The root mean square (r.m.s) delay spread (DS) is the standard deviation value of the delay of multiple paths, and it is weighted proportional to the energy in the reflected waves. In this paper, delay spread is obtained by the same method in [10]. The threshold is given as $20 \mathrm{~dB}$ below the peak level of the power delay profile.

Figure 6(a) shows the results of estimated r.m.s delay spread according to travel distance of RX (meters), and Figure 6(b) shows a cumulative density function (CDF) for statistical distribution of DS. The average (50\% of CDF) is $117 \mathrm{~ns}$, and multiple paths are distributed increasingly with a range from $36 \mathrm{~ns}$ to $404 \mathrm{~ns}$ according to distance.

\section{Angular Spread of Arrival (ASA) and Departure (ASD)}

The r.m.s. angular spread is the power-weighted standard deviation of the direction of arrival and departure, and it is given by the second moment of the power angular profile [5]. In this paper, the threshold level used depends on the dynamic range of channel power (threshold $\mathrm{T}_{h}$ is $20 \mathrm{~dB}$ below the peak level of the angle of power profile at good SNR, threshold $T_{L}$ is $3 \mathrm{~dB}$ over the noise level at low SNR conditions).

Figure 7 and Figure 8 show the results of estimated ASA and ASD, respectively. In Figure 7(a), we can observe that the values of ASA are distributed with a range from $42^{\circ}$ to $100^{\circ}$. Figure 8(a) shows that the values of ASD are spread from $42.2^{\circ}$ to $96^{\circ}$, but it looks getting smaller when the RX is getting more apart from the TX. However, as shown in Figure 7(b) and Figure 8(b), when we compare the average value of ASD with of ASA $\left(85^{\circ}\right.$ in CDF of ASA, $81^{\circ}$ in CDF of ASD), two values are considered to be very similar.

In case of ASD, it looks a larger value when we compared with the ITU-R outdoor channel model for IMT-Advanced system [5]. On urban micro-cell scenario, the ITU-R channel model recommends $25^{\circ}$ as the value of ASD for simulation in the NLoS situation, because the base station considered to be located on the rooftop or below rooftop but much higher than the antenna height of mobile station.

\section{CONCLUSIONS}

From the last research [3], we investigated that the surrounding building heights can affect the propagation characteristics. As the average building height increases, the amount of overall path loss decreases. This can be explained by the virtual waveguide effect, in that the street surrounded with high-rise buildings assumes the role of a waveguide.
In this paper, we performed a channel measurement campaign to observe multipath characteristics for mobile-tomobile direct communications in urban street grid environments, in Seoul, Korea.

Our analysis results from power azimuth spectrum shows that a dominant path group is mostly appeared through the street between high-rise buildings when the RX is not located at corner area. In contrast, at and after corner area, more multipath components (side lobes) which are expected to be reflected from the adjacent buildings could be observed. In case of delay spread, the average value (117 ns) was similar with the value (128 ns) on urban micro-cell scenario of conventional channel model [5]. However, when we compare the result of angular spread of departure with [5], while the value of ASD is $25^{\circ}$ in the NLoS situation, we have the average $81^{\circ}$ as similar as ASA. From this result, we can expect that the conventional MIMO channel model should be improved based on more field measurement data considering the mobile-to-mobile direct communication services.
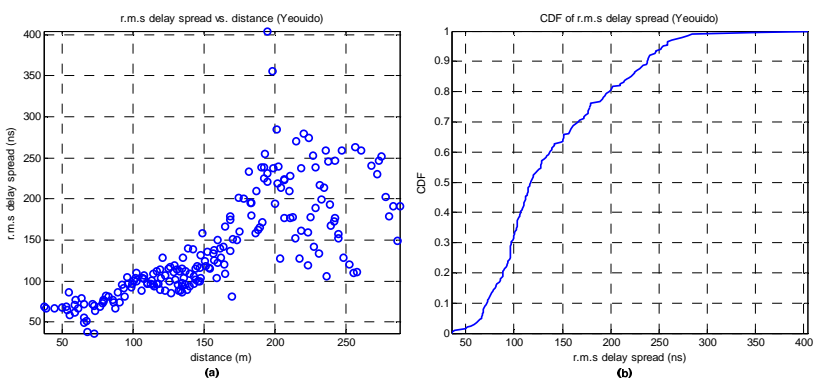

Figure 6. Measurement result: (a) scatter plot of r.m.s deley spread with respect to distance and (b) CDF of r.m.s delay spread
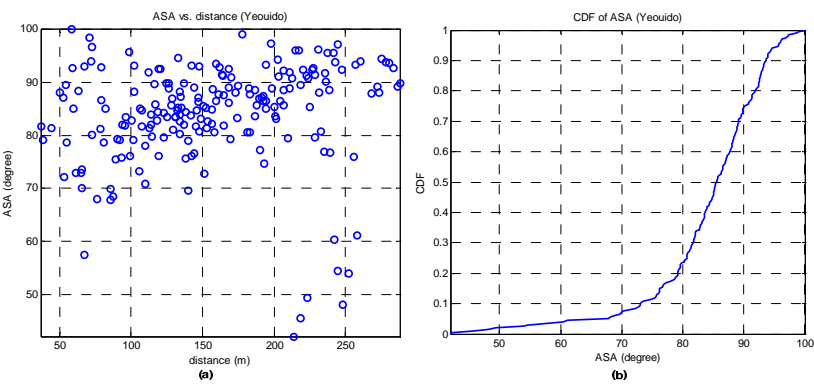

Figure 7. Measurement result: (a) scatter plot of angular spread of arrival with respect to distance and (b) CDF of angular spread of arrival
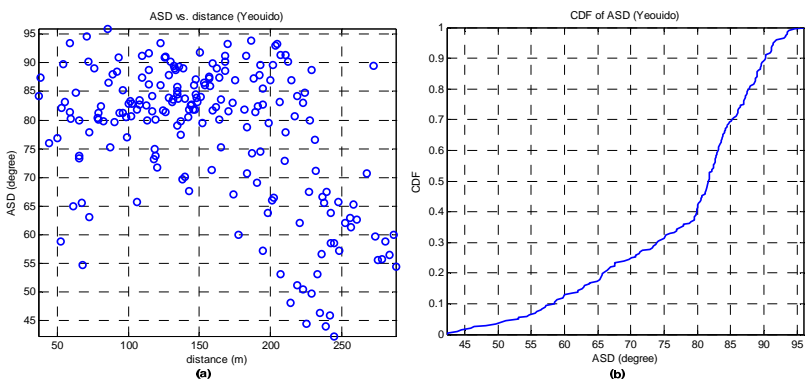

Figure 8. Measurement result: (a) scatter plot of angular spread of departure with respect to distance and (b) CDF of angular spread of departure 


\section{ACKNOWLEDGMENT}

This work was supported by the IT R\&D program of MSIP/KCA, Korea. [2013-005-036-001, Wireless Channel and Frequency Characterization based on Field Measurements for Broadband Mobile Hot-Spot Applications]

\section{REFERENCES}

[1] 3GPP TR 22.803, Feasibility study for Proximity Services (ProSe), Mar. 2013.

[2] J. S. Lu, H. L. Bertoni, C. Chrysanthou, and J. Boksiner, "Simplified path gain model for mobile-to-mobile communications in an urban high-rise environment," in Proc. IEEE Sarnoff Symp., Princeton, NJ, Apr. 2010.

[3] J. Lee, H. K. Chung and M. D. Kim, "Building Height Effects on Path Loss for Low Antenna Links in Urban Street Grid Environments", IEEE Asia Pacific Wireless Communication Symposium, Aug. 2013.

[4] Rec. ITU-R P.1411, Propagation data and prediction methods for the planning of short-range outdoor radiocommunication systems and radio local area networks in the frequency range $300 \mathrm{MHz}$ to $100 \mathrm{GHz}$, Feb. 2012.

[5] Rep. ITU-R M.2135-1, Guidelines for evaluation of radio interface technologies for IMT-Advanced, Dec. 2009.

[6] J.-J. Park, M.-D. Kim, H.-K. Kwon, H.-K. Chung, X. Yin, and Y. Fu, "Measurement-Based Stochastic Cross-Correlation Models of a Multilink Channel in Cooperative Communication Environments," ETRI Journal, vol. 34, pp. 858-868, Dec. 2012.

[7] M. D. Kim, J. J. Park, H. K. Kwon, and H. K. Chung, "Performance Evaluation of Wideband MIMO Relay Channel Sounder for $3.7 \mathrm{GHz}$," IEEE Asia Pacific Wireless Communication Symposium, Aug. 2011.

[8] M. Barlett, "Smoothing periodograms from time series with continuous spectra," Nature (London), vol. 161, no. 8, pp. 686-687, 1948.

[9] H. Chung, N. Vloeberghs, H. K. Kwon, S. J. Lee, and K. C. Lee, "MIMO channel sounder implementation and effects of sounder impairment on statistics of multipath delay spread," Proc. IEEE VTC, vol. 1, pp. 349-353, Sep. 2005.

[10] Rec. ITU-R P.1407, "Multipath propagation and parameterization of its characteristics", Oct. 2009.

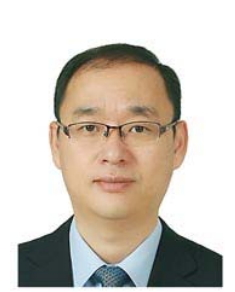

Myung-Don Kim (BS'93-MS'95) is a Principal Researcher in the Advanced Communications Research Laboratory at Electronics and Telecommunications Research Institute (ETRI). He joined ETRI, Daejeon, Rep. of Korea, in 1995, and he worked on the development of mobile test-beds for CDMA, IMT2000 and WCDMA systems. Since 2006, he has been involved in the development of wideband MIMO channel measuring system, measurement and channel estimation of MIMO channels. His research interests include MIMO, channel measurement and channel modeling for next generation mobile communications

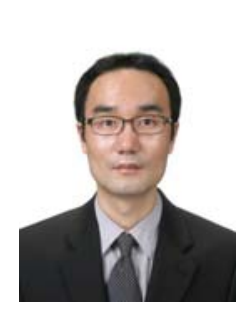

Juyul Lee (BS'96-MS'98-PhD'10) is a Senior Researcher in the Advanced Communications Research Laboratory at Electronics and Telecommunications Research Institute (ETRI) since 2000. Prior joining with ETRI, he was a Research Engineer with the Agency for Defense Development (ADD) from 1998 to 2000. His research spans the fields of information theory and wireless communications, with special interests in multipleantenna/multiple-user/multi-cell resource allocations, device-to-device communications, and wireless propagation channel measurements and modeling.

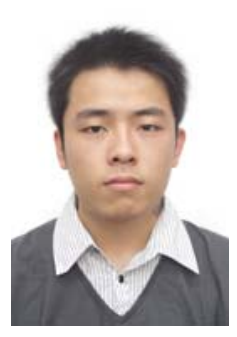

Jinyi Liang (BS'04-MS'13) is a Researcher in the Advanced Communications Research Laboratory at Electronics and Telecommunications Research Institute (ETRI). He is Chinese and joined ETRI, Daejeon, Rep. of Korea, in July 2013, and he's working on the project 'Wireless Channel and Frequency Characterization based on Field

Measurements for Broadband Mobile Hot-Spot Applications'. His research interests include MIMO, channel measurement and channel modeling for next generation mobile communications.

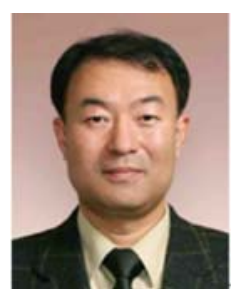

Jinup Kim (BS'85-MS'87-PhD'96) has been with Electronics and Telecommunication Research Institute since 1987. And also he has been a professor of University of Science and Technology in the field of Wireless communications since 2005. He has researched in the field of the wireless communication system. He is recently interested in the Digital RF, channel modeling, Software Defined Radio and Cognitive Radio technologies, etc. 